Acta Crystallographica Section E

Structure Reports

Online

ISSN 1600-5368

\section{$N$-Benzyl-4-methyl-N-(4-methylphenyl)- benzenesulfonamide}

\section{Komal Faryal, ${ }^{\text {a }}$ Muhammad Akhyar Farrukh, ${ }^{\text {}} \neq$ Fahim Ashraf Qureshi, ${ }^{a}$ Saba Ahmad, ${ }^{a}$ Ahmad Adnana and Mehmet Akkurt ${ }^{\text {b* }}$}

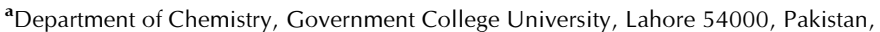
and ${ }^{\mathbf{b}}$ Department of Physics, Faculty of Sciences, Erciyes University, 38039 Kayseri, Turkey

Correspondence e-mail: akkur@@erciyes.edu.tr

Received 14 July 2011; accepted 15 July 2011

Key indicators: single-crystal X-ray study; $T=296 \mathrm{~K}$; mean $\sigma(\mathrm{C}-\mathrm{C})=0.003 \AA$; $R$ factor $=0.047 ; w R$ factor $=0.133 ;$ data-to-parameter ratio $=20.1$.

In the title molecule, $\mathrm{C}_{21} \mathrm{H}_{21} \mathrm{NO}_{2} \mathrm{~S}$, the phenyl ring makes the dihedral angles of $74.13(11)$ and $80.16(11)^{\circ}$ with the two benzene rings, which are inclined at an angle of $43.73(10)^{\circ}$ with respect to each other. In the crystal, molecules are linked by intermolecular $\mathrm{C}-\mathrm{H}$. . O hydrogen bonds along the [010] direction. In addition, a weak $\mathrm{C}-\mathrm{H} \cdots \pi$ (arene) interaction is observed.

\section{Related literature}

For background and the biological and chemical importance of sulfonamides, see: Hung \& Hwang (2007); Burkhart \& Burkhart (2009); Griffiths-Jones et al. (2006). For related structures, see: Ahmad et al. (2011a,b).

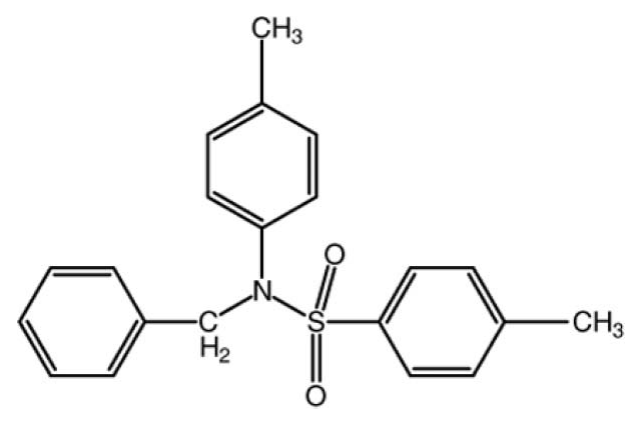

\section{Experimental}

Crystal data

$\mathrm{C}_{21} \mathrm{H}_{21} \mathrm{NO}_{2} \mathrm{~S} \quad M_{r}=351.46$
Monoclinic, $P 2_{1} / c$

$a=9.7089$ (6) А

$b=11.5973(5) \AA$

$c=16.7661(9) \AA$

$\beta=97.691$ (2)

$V=1870.83(17) \AA^{3}$

Data collection

Bruker APEXII CCD

diffractometer

11456 measured reflections

$Z=4$

Mo $K \alpha$ radiation

$\mu=0.19 \mathrm{~mm}^{-1}$

$T=296 \mathrm{~K}$

$0.85 \times 0.13 \times 0.13 \mathrm{~mm}$

Refinement

$R\left[F^{2}>2 \sigma\left(F^{2}\right)\right]=0.047$

$w R\left(F^{2}\right)=0.133$

$S=1.03$

4574 reflections

4574 independent reflections 2964 reflections with $I>2 \sigma(I)$ $R_{\text {int }}=0.023$

228 parameters

$\mathrm{H}$-atom parameters constrained

$\Delta \rho_{\max }=0.20{\mathrm{e} \AA^{-3}}^{-3}$

$\Delta \rho_{\min }=-0.24 \mathrm{e}^{-3}$

Table 1

Hydrogen-bond geometry $\left(\AA{ }^{\circ}\right)$.

$\mathrm{Cg} 2$ is the centroid of the $\mathrm{C} 8-\mathrm{C} 13$ benzene ring.

\begin{tabular}{lllll}
\hline$D-\mathrm{H} \cdots A$ & $D-\mathrm{H}$ & $\mathrm{H} \cdots A$ & $D \cdots A$ & $D-\mathrm{H} \cdots A$ \\
\hline $\mathrm{C} 21-\mathrm{H} 21 \cdots \mathrm{O} 2^{\mathrm{i}}$ & 0.93 & 2.59 & $3.496(2)$ & 166 \\
$\mathrm{C} 14-\mathrm{H} 14 B \cdots C g 2^{\mathrm{ii}}$ & 0.96 & 2.98 & $3.540(2)$ & 118 \\
\hline
\end{tabular}

Symmetry codes: (i) $-x+2, y-\frac{1}{2},-z+\frac{1}{2}$; (ii) $-x+2,-y+1,-z+1$.

Data collection: APEX2 (Bruker, 2007); cell refinement: SAINT (Bruker, 2007); data reduction: $S A I N T$; $\operatorname{program}(\mathrm{s})$ used to solve structure: SHELXS97 (Sheldrick, 2008); program(s) used to refine structure: SHELXL97 (Sheldrick, 2008); molecular graphics: ORTEP-3 for Windows (Farrugia, 1997); software used to prepare material for publication: WinGX (Farrugia, 1999) and PLATON (Spek, 2009).

The authors are grateful to the Higher Education Commission (HEC), Pakistan, for providing funds for the single-crystal XRD facilities at GC University Lahore.

Supplementary data and figures for this paper are available from the IUCr electronic archives (Reference: SI2365).

\title{
References
}

Ahmad, S., Farrukh, M. A., Qureshi, F. A., Adnan, A. \& Akkurt, M. (2011a). Acta Cryst. E67, o303-o304.

Ahmad, S., Farrukh, M. A., Qureshi, F. A., Faryal, K. \& Akkurt, M. (2011b). Acta Cryst. E67, o1909.

Bruker (2007). APEX2 and SAINT. Bruker AXS Inc., Madison, Wisconsin, USA.

Burkhart, C. G. \& Burkhart, C. N. (2009). Open Dermatol. J. 3, 65-67.

Farrugia, L. J. (1997). J. Appl. Cryst. 30, 565.

Farrugia, L. J. (1999). J. Appl. Cryst. 32, 837-838.

Griffiths-Jones, C. M., Hopkin, M. D., Jønsson, D., Ley, V. S., Tapolczay, D. J.,

Vickerstaffe, E. \& Ladlow, M. (2006). J. Comb. Chem. 9, 422-430.

Hung, C. Y. \& Hwang, C. C. (2007). Acta Chromatogr. 18, 106-107.

Sheldrick, G. M. (2008). Acta Cryst. A64, 112-122.

Spek, A. L. (2009). Acta Cryst. D65, 148-155.

¥ Additional correspondence author, e-mail: akhyar@gcu.edu.pk. 


\section{supporting information}

Acta Cryst. (2011). E67, o2100 [doi:10.1107/S1600536811028522]

\section{N-Benzyl-4-methyl-N-(4-methylphenyl)benzenesulfonamide \\ Komal Faryal, Muhammad Akhyar Farrukh, Fahim Ashraf Qureshi, Saba Ahmad, Ahmad Adnan and Mehmet Akkurt}

\section{S1. Comment}

Sulfonamides are compounds of both biological and chemical importance which are widely used in veterinary medicine (Hung \& Hwang, 2007). They were developed in the 1930's and were the first effective antimicrobial agent for systemic administration (Burkhart \& Burkhart, 2009). Sulfonamides represent an important chemotype for medicinal chemistry. They are not only important in drugs with representation of the largest class of antimicrobial agents but also they form the starting point for many classes of drugs including diuretics, antidiabetic drugs, and antihypertensives (Griffiths-Jones et al., 2006). They have bacteriostatic properties and are effective systematic drug used for humans (Ahmad et al., 2011a).

As a contribution to a structural study of sulfonamide derivatives (Ahmad et al., 2011a, Ahmad et al., 2011b), we report here the title compound, $N$-benzyl-4-methyl- $N$-(4-methylphenyl)benzenesulfonamide, (I).

As shown in Fig. 1, the S1 atom in (I) has a distorted tetrahedral geometry. The largest deviation is in the angle O1-S1 $-\mathrm{O} 2\left[120.09(9)^{\circ}\right]$. The phenyl ring $(\mathrm{C} 16-\mathrm{C} 21)$ forms the dihedral angles of $74.13(11)$ and $80.16(11)^{\circ}$ with the two benzene rings ( $\mathrm{C} 1-\mathrm{C} 6$ and $\mathrm{C} 8-\mathrm{C} 13)$. The dihedral angle between the two benzene rings is $43.73(10)^{\circ}$.

In the crystal structure of (I), neighbouring molecules are connected by intermolecular $\mathrm{C}-\mathrm{H} \cdots \mathrm{O}$ hydrogen bonds (Table 1, Fig. 2) along the $b$ axis. The structure is further stabilized by $\mathrm{C}-\mathrm{H} \cdots \pi$ (arene) interactions (Table 1).

\section{S2. Experimental}

$5 \mathrm{~m} M$ of $p$-toluidine was dissolved in $20 \mathrm{ml}$ of distilled water then $5 \mathrm{mM}$ of benzyl chloride was added. The reaction mixture was stirred properly and $5 \mathrm{~m} M$ of $p$-toluenesulfonyl chloride was added. The mixture was stirred for about $1-2 \mathrm{~h}$ and the $\mathrm{pH}$ was maintained $8-10$ using $3 \%$ solution of $\mathrm{Na}_{2} \mathrm{CO}_{3}$. The reaction was monitored by TLC. The product obtained was filtered and the precipitate was washed with distilled water, dried and recrystallized using methanol.

\section{S3. Refinement}

$\mathrm{H}$ atoms were positioned geometrically with $\mathrm{C}-\mathrm{H}=0.93-0.97 \AA$ and allowed to ride on their parent atoms, with $U_{\text {iso }}(\mathrm{H})$ $=1.2$ or $1.5 U_{\text {eq }}(\mathrm{C})$. 


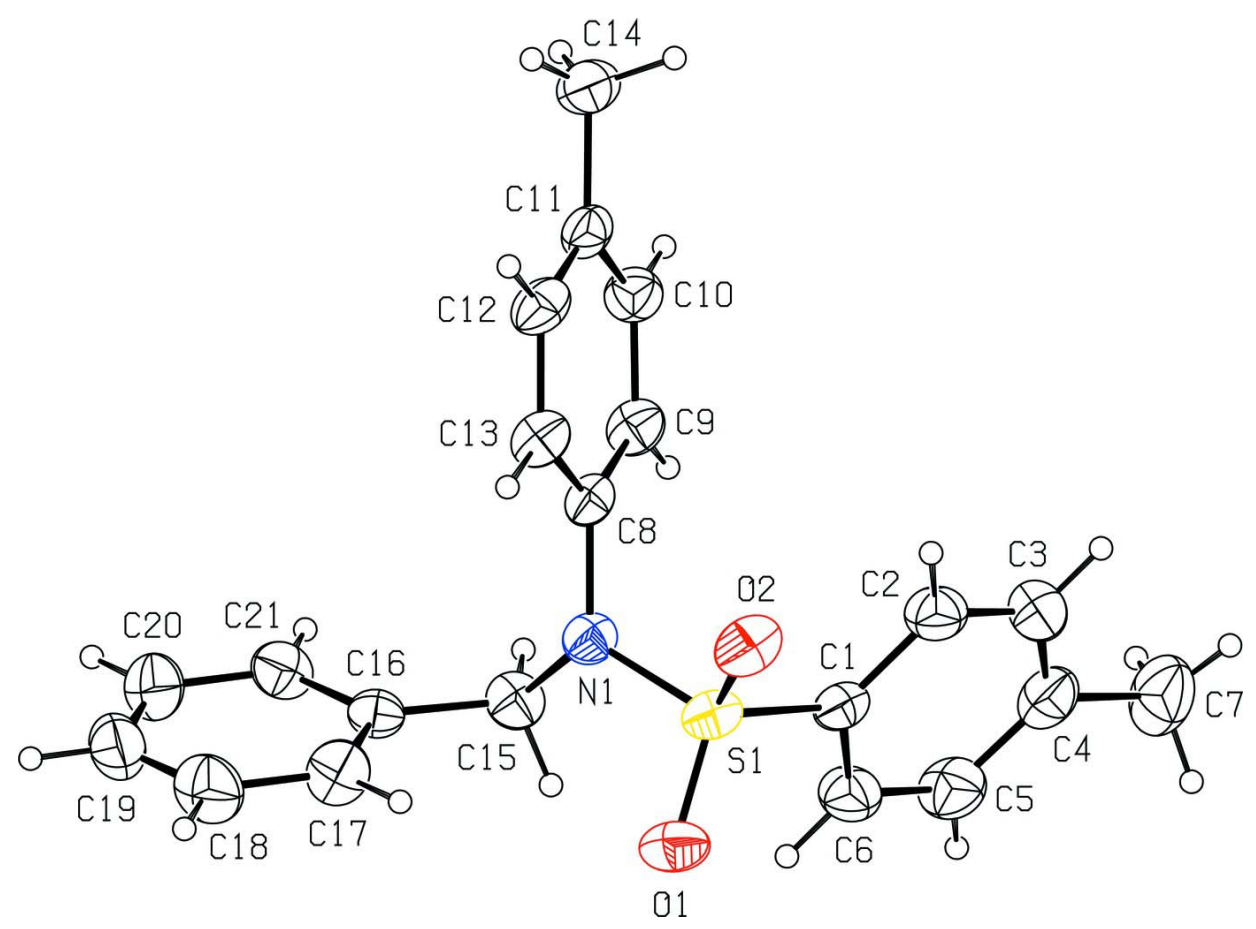

\section{Figure 1}

The molecule of the title compound with the atom numbering scheme. Displacement ellipsoids for non-H atoms are drawn at the $30 \%$ probability level. 


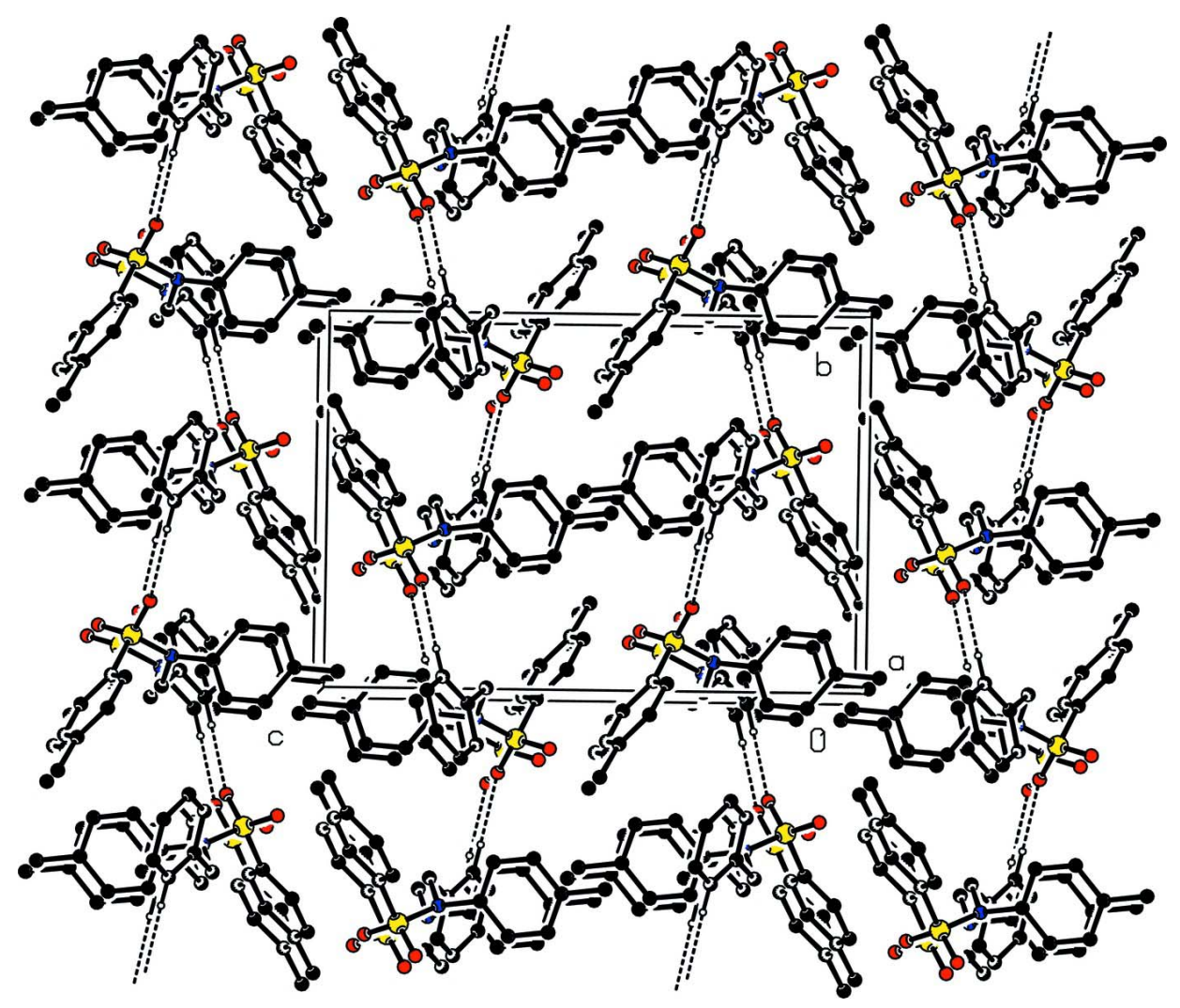

Figure 2

Crystal packing of (I) and the intermolecular $\mathrm{C}-\mathrm{H} \cdots \mathrm{O}$ interactions between the molecules viewed along the $a$ axis. $\mathrm{H}$ atoms not involved in hydrogen bonds (dashed lines) have been omitted for clarity.

\section{N-Benzyl-4-methyl-N-(4-methylphenyl)benzenesulfonamide}

\section{Crystal data}

$\mathrm{C}_{21} \mathrm{H}_{21} \mathrm{NO}_{2} \mathrm{~S}$

$M_{r}=351.46$

Monoclinic, $P 2{ }_{1} / c$

Hall symbol: -P $2 \mathrm{ybc}$

$a=9.7089(6) \AA$

$b=11.5973(5) \AA$

$c=16.7661(9) \AA$

$\beta=97.691(2)^{\circ}$

$V=1870.83(17) \AA^{3}$

$Z=4$

\section{Data collection}

Bruker APEXII CCD

diffractometer

Radiation source: sealed tube

Graphite monochromator

$\varphi$ and $\omega$ scans

11456 measured reflections

4574 independent reflections
$F(000)=744$

$D_{\mathrm{x}}=1.248 \mathrm{Mg} \mathrm{m}^{-3}$

Mo $K \alpha$ radiation, $\lambda=0.71073 \AA$

Cell parameters from 3286 reflections

$\theta=2.5-24.9^{\circ}$

$\mu=0.19 \mathrm{~mm}^{-1}$

$T=296 \mathrm{~K}$

Prism, colourless

$0.85 \times 0.13 \times 0.13 \mathrm{~mm}$

2964 reflections with $I>2 \sigma(I)$

$R_{\text {int }}=0.023$

$\theta_{\text {max }}=28.3^{\circ}, \theta_{\min }=2.9^{\circ}$

$h=-11 \rightarrow 12$

$k=-14 \rightarrow 9$

$l=-22 \rightarrow 22$ 


\section{Refinement}

Refinement on $F^{2}$

Least-squares matrix: full

$R\left[F^{2}>2 \sigma\left(F^{2}\right)\right]=0.047$

$w R\left(F^{2}\right)=0.133$

$S=1.03$

4574 reflections

228 parameters

0 restraints

Primary atom site location: structure-invariant direct methods
Secondary atom site location: difference Fourier map

Hydrogen site location: inferred from neighbouring sites

$\mathrm{H}$-atom parameters constrained

$w=1 /\left[\sigma^{2}\left(F_{\mathrm{o}}^{2}\right)+(0.0588 P)^{2}+0.2963 P\right]$ where $P=\left(F_{\mathrm{o}}{ }^{2}+2 F_{\mathrm{c}}{ }^{2}\right) / 3$

$(\Delta / \sigma)_{\max }<0.001$

$\Delta \rho_{\max }=0.20 \mathrm{e}^{-3}$

$\Delta \rho_{\min }=-0.24$ e $\AA^{-3}$

Special details

Geometry. Bond distances, angles etc. have been calculated using the rounded fractional coordinates. All su's are estimated from the variances of the (full) variance-covariance matrix. The cell e.s.d.'s are taken into account in the estimation of distances, angles and torsion angles

Refinement. Refinement on $F^{2}$ for ALL reflections except those flagged by the user for potential systematic errors. Weighted $R$-factors $w R$ and all goodnesses of fit $S$ are based on $F^{2}$, conventional $R$-factors $R$ are based on $F$, with $F$ set to zero for negative $F^{2}$. The observed criterion of $F^{2}>\sigma\left(F^{2}\right)$ is used only for calculating - $R$-factor-obs etc. and is not relevant to the choice of reflections for refinement. $R$-factors based on $F^{2}$ are statistically about twice as large as those based on $F$, and $R$-factors based on ALL data will be even larger.

Fractional atomic coordinates and isotropic or equivalent isotropic displacement parameters $\left(\AA^{2}\right)$

\begin{tabular}{|c|c|c|c|c|}
\hline & $x$ & $y$ & $z$ & $U_{\text {iso }} * / U_{\text {eq }}$ \\
\hline $\mathrm{S} 1$ & $0.89447(6)$ & $0.62917(4)$ & $0.14182(3)$ & $0.0546(2)$ \\
\hline $\mathrm{O} 1$ & $0.98019(17)$ & $0.65672(12)$ & $0.08157(8)$ & $0.0726(5)$ \\
\hline $\mathrm{O} 2$ & $0.81350(16)$ & $0.71695(11)$ & $0.17304(8)$ & $0.0683(5)$ \\
\hline N1 & $0.99846(16)$ & $0.57740(12)$ & $0.21798(9)$ & $0.0519(5)$ \\
\hline $\mathrm{C} 1$ & $0.7808(2)$ & $0.51881(16)$ & $0.10313(10)$ & $0.0527(6)$ \\
\hline $\mathrm{C} 2$ & $0.6492(2)$ & 0.50937 (19) & $0.12566(13)$ & $0.0687(8)$ \\
\hline $\mathrm{C} 3$ & $0.5614(2)$ & $0.4236(2)$ & $0.09295(14)$ & $0.0780(9)$ \\
\hline $\mathrm{C} 4$ & $0.6002(3)$ & $0.34620(19)$ & $0.03843(13)$ & $0.0733(8)$ \\
\hline $\mathrm{C} 5$ & 0.7323 & $0.3563(2)$ & $0.01758(14)$ & $0.0784(9)$ \\
\hline C6 & $0.8220(2)$ & $0.44090(19)$ & $0.04879(12)$ & $0.0697(8)$ \\
\hline $\mathrm{C} 7$ & 0.5014 & $0.2542(2)$ & $0.00171(18)$ & $0.1051(12)$ \\
\hline $\mathrm{C} 8$ & 0.94537 (19) & $0.56548(14)$ & $0.29392(10)$ & $0.0477(5)$ \\
\hline C9 & $0.8894(2)$ & $0.46311(15)$ & $0.31576(11)$ & $0.0574(6)$ \\
\hline $\mathrm{C} 10$ & $0.8403(2)$ & $0.45394(17)$ & $0.38906(12)$ & $0.0610(7)$ \\
\hline $\mathrm{C} 11$ & $0.8447(2)$ & $0.54630(18)$ & $0.44177(10)$ & $0.0559(6)$ \\
\hline $\mathrm{C} 12$ & $0.9021(2)$ & $0.64773(17)$ & $0.41902(11)$ & $0.0610(7)$ \\
\hline $\mathrm{C} 13$ & $0.9533(2)$ & $0.65776(16)$ & $0.34641(11)$ & $0.0572(6)$ \\
\hline $\mathrm{C} 14$ & $0.7875(2)$ & $0.5353(2)$ & $0.52065(12)$ & $0.0747(8)$ \\
\hline C15 & $1.1138(2)$ & $0.50075(16)$ & $0.20267(12)$ & $0.0583(7)$ \\
\hline $\mathrm{C} 16$ & $1.2518(2)$ & $0.54652(15)$ & $0.23897(11)$ & $0.0501(6)$ \\
\hline $\mathrm{C} 17$ & $1.2928(3)$ & $0.65709(18)$ & $0.22271(14)$ & $0.0722(8)$ \\
\hline $\mathrm{C} 18$ & $1.4232(3)$ & $0.6972(2)$ & $0.25410(17)$ & $0.0871(10)$ \\
\hline C19 & $1.5127(3)$ & $0.6278(3)$ & $0.30155(16)$ & $0.0857(10)$ \\
\hline $\mathrm{C} 20$ & $1.4722(3)$ & 0.5193 & $0.31869(16)$ & $0.0839(10)$ \\
\hline $\mathrm{C} 21$ & $1.3433(2)$ & $0.47864(18)$ & $0.28786(13)$ & $0.0656(8)$ \\
\hline
\end{tabular}




\begin{tabular}{lllll} 
H2 & 0.62040 & 0.56070 & 0.16270 & $0.0820^{*}$ \\
H3 & 0.47300 & 0.41800 & 0.10830 & $0.0940^{*}$ \\
H5 & 0.76130 & 0.30410 & -0.01880 & $0.0940^{*}$ \\
H6 & 0.91030 & 0.44580 & 0.03340 & $0.0840^{*}$ \\
H7A & 0.52650 & 0.18140 & 0.02680 & $0.1580^{*}$ \\
H7B & 0.50670 & 0.24900 & -0.05490 & $0.1580^{*}$ \\
H7C & 0.40830 & 0.27380 & 0.00990 & $0.1580^{*}$ \\
H9 & 0.88460 & 0.40010 & 0.28120 & $0.0690^{*}$ \\
H10 & 0.80330 & 0.38410 & 0.40340 & $0.0730^{*}$ \\
H12 & 0.90650 & 0.71090 & 0.45340 & $0.0730^{*}$ \\
H13 & 0.99310 & 0.72670 & 0.33280 & $0.0690^{*}$ \\
H14A & 0.82210 & 0.59730 & 0.55570 & $0.1120^{*}$ \\
H14B & 0.81600 & 0.46300 & 0.54530 & $0.1120^{*}$ \\
H14C & 0.68780 & 0.53870 & 0.51120 & $0.1120^{*}$ \\
H15A & 1.11300 & 0.49220 & 0.14510 & $0.0700^{*}$ \\
H15B & 1.09980 & 0.42510 & 0.22490 & $0.0700^{*}$ \\
H17 & 1.23230 & 0.70510 & 0.19040 & $0.0870^{*}$ \\
H18 & 1.44980 & 0.77180 & 0.24270 & $0.1040^{*}$ \\
H19 & 1.60070 & 0.65430 & 0.32210 & $0.1030^{*}$ \\
H20 & 1.53270 & 0.47210 & 0.35170 & $0.1010^{*}$ \\
H21 & 1.31760 & 0.40420 & 0.30020 & $0.0790^{*}$ \\
\hline
\end{tabular}

Atomic displacement parameters $\left(\AA^{2}\right)$

\begin{tabular}{lllllll}
\hline & $U^{11}$ & $U^{22}$ & $U^{33}$ & $U^{12}$ & $U^{13}$ & $U^{23}$ \\
\hline S1 & $0.0717(3)$ & $0.0488(3)$ & $0.0443(2)$ & $0.0064(2)$ & $0.0110(2)$ & $0.0024(2)$ \\
O1 & $0.0941(11)$ & $0.0714(9)$ & $0.0561(8)$ & $-0.0079(8)$ & $0.0235(8)$ & $0.0082(7)$ \\
O2 & $0.0904(11)$ & $0.0539(8)$ & $0.0591(8)$ & $0.0215(7)$ & $0.0047(8)$ & $0.0001(6)$ \\
N1 & $0.0557(9)$ & $0.0520(8)$ & $0.0482(8)$ & $0.0090(7)$ & $0.0078(7)$ & $-0.0024(7)$ \\
C1 & $0.0624(12)$ & $0.0568(10)$ & $0.0387(8)$ & $0.0080(9)$ & $0.0062(8)$ & $0.0028(8)$ \\
C2 & $0.0720(15)$ & $0.0785(14)$ & $0.0575(12)$ & $0.0078(12)$ & $0.0162(11)$ & $-0.0034(10)$ \\
C3 & $0.0619(14)$ & $0.0962(17)$ & $0.0762(15)$ & $-0.0046(13)$ & $0.0099(12)$ & $0.0096(14)$ \\
C4 & $0.0850(17)$ & $0.0733(14)$ & $0.0577(12)$ & $-0.0063(13)$ & $-0.0050(12)$ & $0.0072(11)$ \\
C5 & $0.0938(18)$ & $0.0759(15)$ & $0.0663(14)$ & $-0.0070(13)$ & $0.0140(13)$ & $-0.0197(11)$ \\
C6 & $0.0716(14)$ & $0.0768(14)$ & $0.0637(12)$ & $-0.0040(12)$ & $0.0205(11)$ & $-0.0152(11)$ \\
C7 & $0.109(2)$ & $0.099(2)$ & $0.099(2)$ & $-0.0341(17)$ & $-0.0168(17)$ & $0.0030(16)$ \\
C8 & $0.0517(10)$ & $0.0474(9)$ & $0.0421(8)$ & $0.0067(8)$ & $-0.0009(8)$ & $-0.0019(7)$ \\
C9 & $0.0710(13)$ & $0.0470(10)$ & $0.0533(10)$ & $-0.0028(9)$ & $0.0047(10)$ & $-0.0074(8)$ \\
C10 & $0.0676(13)$ & $0.0581(11)$ & $0.0573(11)$ & $-0.0048(10)$ & $0.0082(10)$ & $0.0045(9)$ \\
C11 & $0.0520(11)$ & $0.0688(12)$ & $0.0447(9)$ & $0.0146(10)$ & $-0.0011(8)$ & $0.0039(9)$ \\
C12 & $0.0743(14)$ & $0.0598(12)$ & $0.0472(10)$ & $0.0123(10)$ & $0.0015(10)$ & $-0.0097(8)$ \\
C13 & $0.0732(13)$ & $0.0454(10)$ & $0.0516(10)$ & $0.0031(9)$ & $0.0032(10)$ & $-0.0030(8)$ \\
C14 & $0.0734(15)$ & $0.0983(17)$ & $0.0530(11)$ & $0.0207(13)$ & $0.0112(11)$ & $0.0071(11)$ \\
C15 & $0.0619(13)$ & $0.0487(10)$ & $0.0656(12)$ & $0.0056(9)$ & $0.0132(10)$ & $-0.0092(9)$ \\
C16 & $0.0565(11)$ & $0.0463(9)$ & $0.0497(9)$ & $0.0024(8)$ & $0.0153(9)$ & $-0.0028(8)$ \\
C17 & $0.0826(17)$ & $0.0550(12)$ & $0.0773(14)$ & $-0.0043(11)$ & $0.0041(13)$ & $0.0050(10)$ \\
C18 & $0.093(2)$ & $0.0713(15)$ & $0.0988(19)$ & $-0.0289(15)$ & $0.0195(17)$ & $-0.0106(14)$ \\
C19 & $0.0598(15)$ & $0.114(2)$ & $0.0833(17)$ & $-0.0113(15)$ & $0.0095(13)$ & $-0.0232(16)$ \\
& & & & & &
\end{tabular}


supporting information

\begin{tabular}{lllllll}
$\mathrm{C} 20$ & $0.0652(16)$ & $0.1031(19)$ & $0.0815(16)$ & $0.0104(14)$ & $0.0031(13)$ & $0.0043(14)$ \\
$\mathrm{C} 21$ & $0.0638(14)$ & $0.0626(12)$ & $0.0725(13)$ & $0.0068(11)$ & $0.0168(11)$ & $0.0092(10)$ \\
\hline
\end{tabular}

Geometric parameters $\left(\AA,{ }^{\circ}\right)$

\begin{tabular}{|c|c|c|c|}
\hline $\mathrm{S} 1-\mathrm{O} 1$ & $1.4283(16)$ & $\mathrm{C} 18-\mathrm{C} 19$ & $1.360(4)$ \\
\hline $\mathrm{S} 1-\mathrm{O} 2$ & $1.4278(15)$ & $\mathrm{C} 19-\mathrm{C} 20$ & $1.360(5)$ \\
\hline $\mathrm{S} 1-\mathrm{N} 1$ & $1.6326(16)$ & $\mathrm{C} 20-\mathrm{C} 21$ & $1.372(4)$ \\
\hline $\mathrm{S} 1-\mathrm{C} 1$ & $1.7565(19)$ & $\mathrm{C} 2-\mathrm{H} 2$ & 0.9300 \\
\hline $\mathrm{N} 1-\mathrm{C} 8$ & $1.443(2)$ & $\mathrm{C} 3-\mathrm{H} 3$ & 0.9300 \\
\hline $\mathrm{N} 1-\mathrm{C} 15$ & $1.479(2)$ & $\mathrm{C} 5-\mathrm{H} 5$ & 0.9300 \\
\hline $\mathrm{C} 1-\mathrm{C} 2$ & $1.384(3)$ & $\mathrm{C} 6-\mathrm{H} 6$ & 0.9300 \\
\hline $\mathrm{C} 1-\mathrm{C} 6$ & $1.380(3)$ & C7-H7A & 0.9600 \\
\hline $\mathrm{C} 2-\mathrm{C} 3$ & $1.375(3)$ & $\mathrm{C} 7-\mathrm{H} 7 \mathrm{~B}$ & 0.9600 \\
\hline $\mathrm{C} 3-\mathrm{C} 4$ & $1.369(3)$ & $\mathrm{C} 7-\mathrm{H} 7 \mathrm{C}$ & 0.9600 \\
\hline $\mathrm{C} 4-\mathrm{C} 5$ & $1.379(4)$ & $\mathrm{C} 9-\mathrm{H} 9$ & 0.9300 \\
\hline $\mathrm{C} 4-\mathrm{C} 7$ & $1.510(4)$ & $\mathrm{C} 10-\mathrm{H} 10$ & 0.9300 \\
\hline $\mathrm{C} 5-\mathrm{C} 6$ & $1.369(3)$ & $\mathrm{C} 12-\mathrm{H} 12$ & 0.9300 \\
\hline $\mathrm{C} 8-\mathrm{C} 9$ & $1.376(2)$ & $\mathrm{C} 13-\mathrm{H} 13$ & 0.9300 \\
\hline $\mathrm{C} 8-\mathrm{C} 13$ & $1.381(2)$ & $\mathrm{C} 14-\mathrm{H} 14 \mathrm{~A}$ & 0.9600 \\
\hline $\mathrm{C} 9-\mathrm{C} 10$ & $1.381(3)$ & C14-H14B & 0.9600 \\
\hline $\mathrm{C} 10-\mathrm{C} 11$ & $1.386(3)$ & $\mathrm{C} 14-\mathrm{H} 14 \mathrm{C}$ & 0.9600 \\
\hline $\mathrm{C} 11-\mathrm{C} 12$ & $1.377(3)$ & C15-H15A & 0.9700 \\
\hline $\mathrm{C} 11-\mathrm{C} 14$ & $1.507(3)$ & C15-H15B & 0.9700 \\
\hline $\mathrm{C} 12-\mathrm{C} 13$ & $1.380(3)$ & C17-H17 & 0.9300 \\
\hline $\mathrm{C} 15-\mathrm{C} 16$ & $1.493(3)$ & $\mathrm{C} 18-\mathrm{H} 18$ & 0.9300 \\
\hline $\mathrm{C} 16-\mathrm{C} 17$ & $1.381(3)$ & $\mathrm{C} 19-\mathrm{H} 19$ & 0.9300 \\
\hline $\mathrm{C} 16-\mathrm{C} 21$ & $1.373(3)$ & $\mathrm{C} 20-\mathrm{H} 20$ & 0.9300 \\
\hline $\mathrm{C} 17-\mathrm{C} 18$ & $1.385(4)$ & $\mathrm{C} 21-\mathrm{H} 21$ & 0.9300 \\
\hline $\mathrm{O} 1-\mathrm{S} 1-\mathrm{O} 2$ & $120.09(9)$ & $\mathrm{C} 4-\mathrm{C} 3-\mathrm{H} 3$ & 119.00 \\
\hline $\mathrm{O} 1-\mathrm{S} 1-\mathrm{N} 1$ & $106.11(9)$ & $\mathrm{C} 4-\mathrm{C} 5-\mathrm{H} 5$ & 119.00 \\
\hline $\mathrm{O} 1-\mathrm{S} 1-\mathrm{C} 1$ & $107.37(9)$ & $\mathrm{C} 6-\mathrm{C} 5-\mathrm{H} 5$ & 119.00 \\
\hline $\mathrm{O} 2-\mathrm{S} 1-\mathrm{N} 1$ & $106.78(8)$ & $\mathrm{C} 1-\mathrm{C} 6-\mathrm{H} 6$ & 120.00 \\
\hline $\mathrm{O} 2-\mathrm{S} 1-\mathrm{C} 1$ & $107.78(9)$ & $\mathrm{C} 5-\mathrm{C} 6-\mathrm{H} 6$ & 120.00 \\
\hline $\mathrm{N} 1-\mathrm{S} 1-\mathrm{C} 1$ & $108.25(8)$ & $\mathrm{C} 4-\mathrm{C} 7-\mathrm{H} 7 \mathrm{~A}$ & 109.00 \\
\hline $\mathrm{S} 1-\mathrm{N} 1-\mathrm{C} 8$ & $117.96(12)$ & $\mathrm{C} 4-\mathrm{C} 7-\mathrm{H} 7 \mathrm{~B}$ & 109.00 \\
\hline $\mathrm{S} 1-\mathrm{N} 1-\mathrm{C} 15$ & $119.26(12)$ & $\mathrm{C} 4-\mathrm{C} 7-\mathrm{H} 7 \mathrm{C}$ & 109.00 \\
\hline $\mathrm{C} 8-\mathrm{N} 1-\mathrm{C} 15$ & $117.64(14)$ & $\mathrm{H} 7 \mathrm{~A}-\mathrm{C} 7-\mathrm{H} 7 \mathrm{~B}$ & 110.00 \\
\hline $\mathrm{S} 1-\mathrm{C} 1-\mathrm{C} 2$ & $120.94(15)$ & $\mathrm{H} 7 \mathrm{~A}-\mathrm{C} 7-\mathrm{H} 7 \mathrm{C}$ & 110.00 \\
\hline $\mathrm{S} 1-\mathrm{C} 1-\mathrm{C} 6$ & $119.84(15)$ & $\mathrm{H} 7 \mathrm{~B}-\mathrm{C} 7-\mathrm{H} 7 \mathrm{C}$ & 109.00 \\
\hline $\mathrm{C} 2-\mathrm{C} 1-\mathrm{C} 6$ & $119.22(18)$ & $\mathrm{C} 8-\mathrm{C} 9-\mathrm{H} 9$ & 120.00 \\
\hline $\mathrm{C} 1-\mathrm{C} 2-\mathrm{C} 3$ & $119.59(19)$ & $\mathrm{C} 10-\mathrm{C} 9-\mathrm{H} 9$ & 120.00 \\
\hline $\mathrm{C} 2-\mathrm{C} 3-\mathrm{C} 4$ & $121.9(2)$ & $\mathrm{C} 9-\mathrm{C} 10-\mathrm{H} 10$ & 119.00 \\
\hline $\mathrm{C} 3-\mathrm{C} 4-\mathrm{C} 5$ & $117.5(2)$ & $\mathrm{C} 11-\mathrm{C} 10-\mathrm{H} 10$ & 119.00 \\
\hline $\mathrm{C} 3-\mathrm{C} 4-\mathrm{C} 7$ & $121.3(2)$ & $\mathrm{C} 11-\mathrm{C} 12-\mathrm{H} 12$ & 119.00 \\
\hline $\mathrm{C} 5-\mathrm{C} 4-\mathrm{C} 7$ & $121.2(2)$ & $\mathrm{C} 13-\mathrm{C} 12-\mathrm{H} 12$ & 119.00 \\
\hline $\mathrm{C} 4-\mathrm{C} 5-\mathrm{C} 6$ & $122.0(2)$ & $\mathrm{C} 8-\mathrm{C} 13-\mathrm{H} 13$ & 120.00 \\
\hline
\end{tabular}




\begin{tabular}{|c|c|c|c|}
\hline $\mathrm{C} 1-\mathrm{C} 6-\mathrm{C} 5$ & $119.7(2)$ & $\mathrm{C} 12-\mathrm{C} 13-\mathrm{H} 13$ & 120.00 \\
\hline $\mathrm{N} 1-\mathrm{C} 8-\mathrm{C} 9$ & $121.21(15)$ & $\mathrm{C} 11-\mathrm{C} 14-\mathrm{H} 14 \mathrm{~A}$ & 110.00 \\
\hline $\mathrm{N} 1-\mathrm{C} 8-\mathrm{C} 13$ & $119.47(15)$ & $\mathrm{C} 11-\mathrm{C} 14-\mathrm{H} 14 \mathrm{~B}$ & 109.00 \\
\hline $\mathrm{C} 9-\mathrm{C} 8-\mathrm{C} 13$ & $119.31(16)$ & $\mathrm{C} 11-\mathrm{C} 14-\mathrm{H} 14 \mathrm{C}$ & 109.00 \\
\hline $\mathrm{C} 8-\mathrm{C} 9-\mathrm{C} 10$ & $119.95(17)$ & $\mathrm{H} 14 \mathrm{~A}-\mathrm{C} 14-\mathrm{H} 14 \mathrm{~B}$ & 109.00 \\
\hline $\mathrm{C} 9-\mathrm{C} 10-\mathrm{C} 11$ & $121.56(18)$ & $\mathrm{H} 14 \mathrm{~A}-\mathrm{C} 14-\mathrm{H} 14 \mathrm{C}$ & 109.00 \\
\hline $\mathrm{C} 10-\mathrm{C} 11-\mathrm{C} 12$ & $117.56(17)$ & $\mathrm{H} 14 \mathrm{~B}-\mathrm{C} 14-\mathrm{H} 14 \mathrm{C}$ & 109.00 \\
\hline $\mathrm{C} 10-\mathrm{C} 11-\mathrm{C} 14$ & $120.72(18)$ & $\mathrm{N} 1-\mathrm{C} 15-\mathrm{H} 15 \mathrm{~A}$ & 109.00 \\
\hline $\mathrm{C} 12-\mathrm{C} 11-\mathrm{C} 14$ & $121.72(18)$ & $\mathrm{N} 1-\mathrm{C} 15-\mathrm{H} 15 \mathrm{~B}$ & 109.00 \\
\hline $\mathrm{C} 11-\mathrm{C} 12-\mathrm{C} 13$ & $121.55(18)$ & $\mathrm{C} 16-\mathrm{C} 15-\mathrm{H} 15 \mathrm{~A}$ & 109.00 \\
\hline $\mathrm{C} 8-\mathrm{C} 13-\mathrm{C} 12$ & $120.05(17)$ & $\mathrm{C} 16-\mathrm{C} 15-\mathrm{H} 15 \mathrm{~B}$ & 109.00 \\
\hline $\mathrm{N} 1-\mathrm{C} 15-\mathrm{C} 16$ & $112.00(15)$ & $\mathrm{H} 15 \mathrm{~A}-\mathrm{C} 15-\mathrm{H} 15 \mathrm{~B}$ & 108.00 \\
\hline $\mathrm{C} 15-\mathrm{C} 16-\mathrm{C} 17$ & $121.09(19)$ & $\mathrm{C} 16-\mathrm{C} 17-\mathrm{H} 17$ & 120.00 \\
\hline $\mathrm{C} 15-\mathrm{C} 16-\mathrm{C} 21$ & $120.87(17)$ & $\mathrm{C} 18-\mathrm{C} 17-\mathrm{H} 17$ & 120.00 \\
\hline $\mathrm{C} 17-\mathrm{C} 16-\mathrm{C} 21$ & $118.0(2)$ & $\mathrm{C} 17-\mathrm{C} 18-\mathrm{H} 18$ & 120.00 \\
\hline $\mathrm{C} 16-\mathrm{C} 17-\mathrm{C} 18$ & $120.7(2)$ & $\mathrm{C} 19-\mathrm{C} 18-\mathrm{H} 18$ & 120.00 \\
\hline $\mathrm{C} 17-\mathrm{C} 18-\mathrm{C} 19$ & $120.2(2)$ & $\mathrm{C} 18-\mathrm{C} 19-\mathrm{H} 19$ & 120.00 \\
\hline $\mathrm{C} 18-\mathrm{C} 19-\mathrm{C} 20$ & $119.4(3)$ & $\mathrm{C} 20-\mathrm{C} 19-\mathrm{H} 19$ & 120.00 \\
\hline $\mathrm{C} 19-\mathrm{C} 20-\mathrm{C} 21$ & $120.9(3)$ & $\mathrm{C} 19-\mathrm{C} 20-\mathrm{H} 20$ & 120.00 \\
\hline $\mathrm{C} 16-\mathrm{C} 21-\mathrm{C} 20$ & $120.8(2)$ & $\mathrm{C} 21-\mathrm{C} 20-\mathrm{H} 20$ & 120.00 \\
\hline $\mathrm{C} 1-\mathrm{C} 2-\mathrm{H} 2$ & 120.00 & $\mathrm{C} 16-\mathrm{C} 21-\mathrm{H} 21$ & 120.00 \\
\hline $\mathrm{C} 3-\mathrm{C} 2-\mathrm{H} 2$ & 120.00 & $\mathrm{C} 20-\mathrm{C} 21-\mathrm{H} 21$ & 120.00 \\
\hline $\mathrm{C} 2-\mathrm{C} 3-\mathrm{H} 3$ & 119.00 & & \\
\hline $\mathrm{O} 1-\mathrm{S} 1-\mathrm{N} 1-\mathrm{C} 8$ & $166.82(12)$ & $\mathrm{C} 2-\mathrm{C} 3-\mathrm{C} 4-\mathrm{C} 5$ & $0.6(3)$ \\
\hline $\mathrm{O} 2-\mathrm{S} 1-\mathrm{N} 1-\mathrm{C} 8$ & $37.62(14)$ & $\mathrm{C} 7-\mathrm{C} 4-\mathrm{C} 5-\mathrm{C} 6$ & $178.4(2)$ \\
\hline $\mathrm{C} 1-\mathrm{S} 1-\mathrm{N} 1-\mathrm{C} 8$ & $-78.19(14)$ & $\mathrm{C} 3-\mathrm{C} 4-\mathrm{C} 5-\mathrm{C} 6$ & $-0.9(3)$ \\
\hline $\mathrm{O} 1-\mathrm{S} 1-\mathrm{N} 1-\mathrm{C} 15$ & $-39.11(15)$ & $\mathrm{C} 4-\mathrm{C} 5-\mathrm{C} 6-\mathrm{C} 1$ & $0.3(3)$ \\
\hline $\mathrm{O} 2-\mathrm{S} 1-\mathrm{N} 1-\mathrm{C} 15$ & $-168.32(13)$ & $\mathrm{N} 1-\mathrm{C} 8-\mathrm{C} 13-\mathrm{C} 12$ & $179.59(17)$ \\
\hline $\mathrm{C} 1-\mathrm{S} 1-\mathrm{N} 1-\mathrm{C} 15$ & $75.88(15)$ & $\mathrm{N} 1-\mathrm{C} 8-\mathrm{C} 9-\mathrm{C} 10$ & $179.53(17)$ \\
\hline $\mathrm{O} 1-\mathrm{S} 1-\mathrm{C} 1-\mathrm{C} 2$ & $-149.26(16)$ & $\mathrm{C} 13-\mathrm{C} 8-\mathrm{C} 9-\mathrm{C} 10$ & $0.8(3)$ \\
\hline $\mathrm{O} 2-\mathrm{S} 1-\mathrm{C} 1-\mathrm{C} 2$ & $-18.57(18)$ & $\mathrm{C} 9-\mathrm{C} 8-\mathrm{C} 13-\mathrm{C} 12$ & $-1.7(3)$ \\
\hline $\mathrm{N} 1-\mathrm{S} 1-\mathrm{C} 1-\mathrm{C} 2$ & $96.58(17)$ & $\mathrm{C} 8-\mathrm{C} 9-\mathrm{C} 10-\mathrm{C} 11$ & $0.5(3)$ \\
\hline $\mathrm{O} 1-\mathrm{S} 1-\mathrm{C} 1-\mathrm{C} 6$ & $30.00(18)$ & $\mathrm{C} 9-\mathrm{C} 10-\mathrm{C} 11-\mathrm{C} 14$ & $178.56(18)$ \\
\hline $\mathrm{O} 2-\mathrm{S} 1-\mathrm{C} 1-\mathrm{C} 6$ & $160.69(15)$ & $\mathrm{C} 9-\mathrm{C} 10-\mathrm{C} 11-\mathrm{C} 12$ & $-1.0(3)$ \\
\hline $\mathrm{N} 1-\mathrm{S} 1-\mathrm{C} 1-\mathrm{C} 6$ & $-84.16(17)$ & $\mathrm{C} 10-\mathrm{C} 11-\mathrm{C} 12-\mathrm{C} 13$ & $0.1(3)$ \\
\hline $\mathrm{S} 1-\mathrm{N} 1-\mathrm{C} 8-\mathrm{C} 9$ & $94.38(18)$ & $\mathrm{C} 14-\mathrm{C} 11-\mathrm{C} 12-\mathrm{C} 13$ & $-179.43(18)$ \\
\hline $\mathrm{C} 15-\mathrm{N} 1-\mathrm{C} 8-\mathrm{C} 9$ & $-60.1(2)$ & $\mathrm{C} 11-\mathrm{C} 12-\mathrm{C} 13-\mathrm{C} 8$ & $1.2(3)$ \\
\hline $\mathrm{S} 1-\mathrm{N} 1-\mathrm{C} 15-\mathrm{C} 16$ & $122.31(15)$ & $\mathrm{N} 1-\mathrm{C} 15-\mathrm{C} 16-\mathrm{C} 17$ & $-53.5(2)$ \\
\hline $\mathrm{C} 15-\mathrm{N} 1-\mathrm{C} 8-\mathrm{C} 13$ & $118.60(19)$ & $\mathrm{N} 1-\mathrm{C} 15-\mathrm{C} 16-\mathrm{C} 21$ & $128.29(19)$ \\
\hline $\mathrm{S} 1-\mathrm{N} 1-\mathrm{C} 8-\mathrm{C} 13$ & $-86.91(19)$ & $\mathrm{C} 15-\mathrm{C} 16-\mathrm{C} 17-\mathrm{C} 18$ & $-177.4(2)$ \\
\hline $\mathrm{C} 8-\mathrm{N} 1-\mathrm{C} 15-\mathrm{C} 16$ & $-83.55(19)$ & $\mathrm{C} 21-\mathrm{C} 16-\mathrm{C} 17-\mathrm{C} 18$ & $0.9(3)$ \\
\hline $\mathrm{C} 6-\mathrm{C} 1-\mathrm{C} 2-\mathrm{C} 3$ & $-0.7(3)$ & $\mathrm{C} 15-\mathrm{C} 16-\mathrm{C} 21-\mathrm{C} 20$ & $177.5(2)$ \\
\hline $\mathrm{S} 1-\mathrm{C} 1-\mathrm{C} 2-\mathrm{C} 3$ & $178.54(17)$ & $\mathrm{C} 17-\mathrm{C} 16-\mathrm{C} 21-\mathrm{C} 20$ & $-0.8(3)$ \\
\hline $\mathrm{C} 2-\mathrm{C} 1-\mathrm{C} 6-\mathrm{C} 5$ & $0.5(3)$ & $\mathrm{C} 16-\mathrm{C} 17-\mathrm{C} 18-\mathrm{C} 19$ & $0.0(4)$ \\
\hline $\mathrm{S} 1-\mathrm{C} 1-\mathrm{C} 6-\mathrm{C} 5$ & $-178.78(17)$ & $\mathrm{C} 17-\mathrm{C} 18-\mathrm{C} 19-\mathrm{C} 20$ & $-0.9(4)$ \\
\hline $\mathrm{C} 1-\mathrm{C} 2-\mathrm{C} 3-\mathrm{C} 4$ & $0.2(3)$ & $\mathrm{C} 18-\mathrm{C} 19-\mathrm{C} 20-\mathrm{C} 21$ & $0.9(4)$ \\
\hline $\mathrm{C} 2-\mathrm{C} 3-\mathrm{C} 4-\mathrm{C} 7$ & $-178.6(2)$ & $\mathrm{C} 19-\mathrm{C} 20-\mathrm{C} 21-\mathrm{C} 16$ & $-0.1(4)$ \\
\hline
\end{tabular}




\section{supporting information}

Hydrogen-bond geometry $\left(A,{ }^{\circ}\right)$

$\mathrm{Cg} 2$ is the centroid of the $\mathrm{C} 8-\mathrm{C} 13$ benzene ring.

\begin{tabular}{lllll}
\hline$D-\mathrm{H} \cdots A$ & $D-\mathrm{H}$ & $\mathrm{H} \cdots A$ & $D \cdots A$ & $D-\mathrm{H} \cdots A$ \\
\hline $\mathrm{C} 2-\mathrm{H} 2 \cdots \mathrm{O} 2$ & 0.93 & 2.60 & $2.938(3)$ & 102 \\
$\mathrm{C} 15-\mathrm{H} 15 A \cdots \mathrm{O} 1$ & 0.97 & 2.46 & $2.894(2)$ & 107 \\
$\mathrm{C} 21-\mathrm{H} 21 \cdots \mathrm{O} 2^{\mathrm{i}}$ & 0.93 & 2.59 & $3.496(2)$ & 166 \\
$\mathrm{C} 14-\mathrm{H} 14 B \cdots C g 2^{\mathrm{ii}}$ & 0.96 & 2.98 & $3.540(2)$ & 118 \\
\hline
\end{tabular}

Symmetry codes: (i) $-x+2, y-1 / 2,-z+1 / 2$; (ii) $-x+2,-y+1,-z+1$. 